\title{
Accuracy of transcranial ultrasound and color duplex in diagnosis of neonatal brain pathologies
}

Sara Ahmed Mohamed Ahmed ${ }^{a}$, Ahmed Hesham Said ${ }^{b}$ and Mohamed Ahmed Mohamed Soliman ${ }^{b}$

${ }^{a}$ Radiodiagnosis department, Faculty of Medicine, Beni-Suef University, Egypt

\begin{abstract}
The aim of the study: was to document the value of transcranial ultrasound and color duplex in detection of abnormalities in neonatal brain. This is cross sectional study was performed on 40 patients in neonates group with neurological sign or suspected sign for neurological lesion. All cases had cranial ultrasound. Our study was conducted from May 2016 to March 2017, in the Department of Radiodiagnosis, at Beni Suef university hospital ,Patients were all selected from Benisuef university hospital; either picked up from the outpatient clinics or from the inpatient wards of neonatology unit and ICU unit . All the studied cases were subjected to: full history taking, full clinical Examination, investigations and transcranial ultrasound examination. This study showed that the Ultra-sound diagnosed $10(25 \%)$ with normal brain and $30(75 \%)$ with abnormal CUS findings. Among the abnormal CUS findings, 5(16.6\%) with congenital abnormalities, 25(83.4\%) with acquired lesions.
\end{abstract}

Keywords: Trans cranial ultrasound, trans cranial doppler. 
(US) study that involves the use of a low frequency $(\leq 2 \mathrm{MHz})$ transducer probe to insonate the basal cerebral arteries through relatively thin bone windows. TCD allows dynamic monitoring of cerebral blood flow velocity $(\mathrm{CBF}-\mathrm{V})$ and vessel pulsatility over extended periods with a high temporal .resolution (4)

Recent advances in sonographic technology have made ultrasound an increasingly accurate and adequate means to detect cranial morphology and intracranial pathology in infants. When modern equipment is combined with thorough imaging technique, ultrasound delivers similar results to MRI in terms of sensitivity and ability to direct initial management Cranial sonography is less expensive, spares the patient from radiation, does not require sedation, and its portability allows for bedside evaluation in gravely ill infants who cannot be transported to radiology .for imaging (5)

\section{Patients and Methods .2}

This is cross sectional study was performed on 40 patients in neonates group with neurological sign or suspected sign for neurological lesion. All cases had cranial .ultrasound

\section{:Inclusion criteria 2.1}

Full term or preterm infants clinically presented with neurological signs e.g. (convulsions ,enlarged head, pathological jaundice ,trauma, Obstructed labor, bleeding

\section{Introduction .1}

The fetal or preterm infant's brain is vulnerable to both hemorrhagic and ischemic injuries during the late second and early third trimesters. This is due to vascular, cellular and anatomical features of the developing brain, and the tendency for preterm infants to experience periods of physiological instability at a time when they have limited cerebral .circulatory auto regulation (1)

Several problems are associated with imaging of critically ill neonates. These include the choice of imaging technique, the timing of the imaging study, and transporting acutely ill neonates, many of whom require ventilator assistance and multiple indwelling catheters. As a result, there is a great need to a reliable tool for detecting congenital and acquired anomalies of the perinatal brain and the most frequently occurring patterns of brain injury in both preterm and full-term neonates

Cranial ultrasonography (CUS) was introduced into neonatology in the late 1970 s and has become an essential diagnostic tool in modern neonatology. The non-invasive nature of ultrasonography makes it an ideal imaging technique in the neonates. The fontanels and many sutures of the skull are still open, and these can be used as acoustic windows to ."look" into the brain (3)

Trans Cranial Doppler (TCD), first described in 1982, is a noninvasive ultrasound 
Device: All patients were examined using xario200 ultrasound device (TOSHIBA health care) that is equipped by convex probe, linear .probe and microconvex probe

\section{:Technique of examination}

\section{A. Transcranial Ultrasonography}

Patient position: The patient lies supine .1 with lateral tilting of his head to either side during most of the examination except scanning through the foramen magnum during which the patient is sitting with slight flexion .of his head forwards Anterior fontanel: The anterior fontanel was .2 used as the principal acoustic window using convex probe, and the scanning procedures :included

Six standard coronal planes frontal lobes, frontal horns of the lateral Ventricles, foramen of Monro and the third ventricle, body of the lateral Ventricles, trigon of the lateral ventricle, and occipital lobes. Five standard Sagittal planes mid sagittal plane, left and right Parasagittal planes through lateral ventricles, .right and left parasagittal Planes Temporal Window: Scanning through this .3 approach was mainly used for transcranial Doppler examination of the middle cerebral artery in some cases and more clear view of .the temporal lobe Posterolateral (mastoid) fontanel: Images .4 of the posterior fossa were obtained through the posterolateral fontanels. The convex transducer was placed over the posterolateral fontanels. The transducer was then rotated tendency, multiple congenital anomalies, respiratory distress ,physiological jaundice ,persistent vomiting).

\section{:Exclusion criteria 2.2}

Full term or preterm infant with no .1 .neurological sign or symptoms

.Still birth .2

Patients were subjected to the following:

\section{:Clinical assessment} Gestational age, weeks (mean _ SD) Body weight, g Length at birth, $\mathrm{cm}$ Head circumference at birth, $\mathrm{cm}$ Premature rupture of membranes Mode of delivery: normal vaginal delivery/cesarean section

Gender

Antenatal steroids given for mother Vitamin $\mathrm{K}$ given for infant ,Resuscitated at birth (bag/mask or Neopuff) ,Intubated at birth -

Initial diagnosis of perinatal asphyxia Respiratory distress/congenital pneumonia Infants treated only with Continuous .positive air way pressure (CPAP) Infants need to mechanical ventilation Received blood transfusion Vital signs (pulse\& respiratory rate) :Imaging investigations All patients were subjected to Transcranial ultrasound examination Methods: All sonographic examinations were performed at department of radio diagnosis at .Beni Suef university hospital 
RI during the first year of life. After fontanelle closure, the mean RI decreases to .between 0.50 and 0.60 (6)

\section{Statistical methodology}

- Analysis of data was done by IBM computer using SPSS (statistical program for social science) as follows;

- Description of quantitative variables as mean, $\mathrm{SD}$ and range.

- Description of qualitative variables as number and percentage.

- Unpaired t-test was used to compare quantitative variables, in parametric data $(\mathrm{SD}<$ $50 \%$ mean)

- $\mathrm{P}$ value $>0.05$ insignificant

$\cdot \mathrm{P}<0.05$ significant

- $\mathrm{P}<0.01$ highly significant [20]. slightly using real-time imaging to obtain the appropriate angled axial image depicting the cisterna magna, cerebellar hemispheres, vermis .cerebelli, fourth ventricle and pons Posterior Fontanel: The posterior fontanel .5 was palpated and the convex transducer placed in the middle of the fontanel, with the marker in the horizontal position to obtain a coronal plane and in the vertical position to obtain a sagittal plane. Scanning through the posterior fontanel was of a high value mainly in assessing the posterior fossa in newborn patients with meningeo-myelocele and .suspected to have Chiari malformation

\section{B. Transcranial Doppler Examination}

Transcranial color coded Doppler was especially applied to assess vascular lesions in all examined patients. By scanning through the anterior fontanel to image, flow in the circle of Willis in both coronal and sagittal scans using convex probe. The MCA can be assessed in its echogenic fissure in the axial plane through temporal approach using linear probe. RI was .calculated for MCA\&ACA Resistance index (RI) is peak systolic flow velocity (PSV) minus end diastolic velocity (EDV) divided by (PSV). The overall mean RI of the major intracranial vessels namely ICA, ACA and MCA in normal term neonates is 0.726. There is a known downward trend of the 
Results .3

Table (1) showed that a total number of 40 neonates under the study with minimum age 30 weeks and maximum 40 weeks with mean age 37.65 weeks, minimum birth weight 1100 grams and maximum 3500 grams with mean 2847.50. Minimum Length at Birth $(\mathrm{cm})$ and Head circumference at Birth $(\mathrm{cm})$ of $(44 \mathrm{~cm}, 33 \mathrm{~cm})$ and maximum of $(52 \mathrm{~cm}, 55 \mathrm{~cm})$ with mean 48.25 and 39.05 respectively.

Table (1): Description of Gestational Age, Birth Weight, Length and Head Circumference at Birth and time of examination of Neonates under the Study:

\begin{tabular}{|l|l|l|l|l|l|}
\hline Parameter & N & Min & Max & Mean & Std. Dev \\
\hline Gestational Age (w) & 40 & 30.00 & 40.00 & 37.650 & 1.889 \\
\hline Birth Weight (gm) & 40 & 1100 & 3500 & 2847.50 & 540.056 \\
\hline Length at Birth (cm) & 40 & 44.00 & 52.00 & 48.25 & 2.509 \\
\hline Head circumference at Birth $(\mathrm{cm})$ & 40 & 33.00 & 55.00 & 39.05 & 6.341 \\
\hline Time of examination (day) & 40 & 2.00 & 27.00 & 15.48 & 7.547 \\
\hline
\end{tabular}

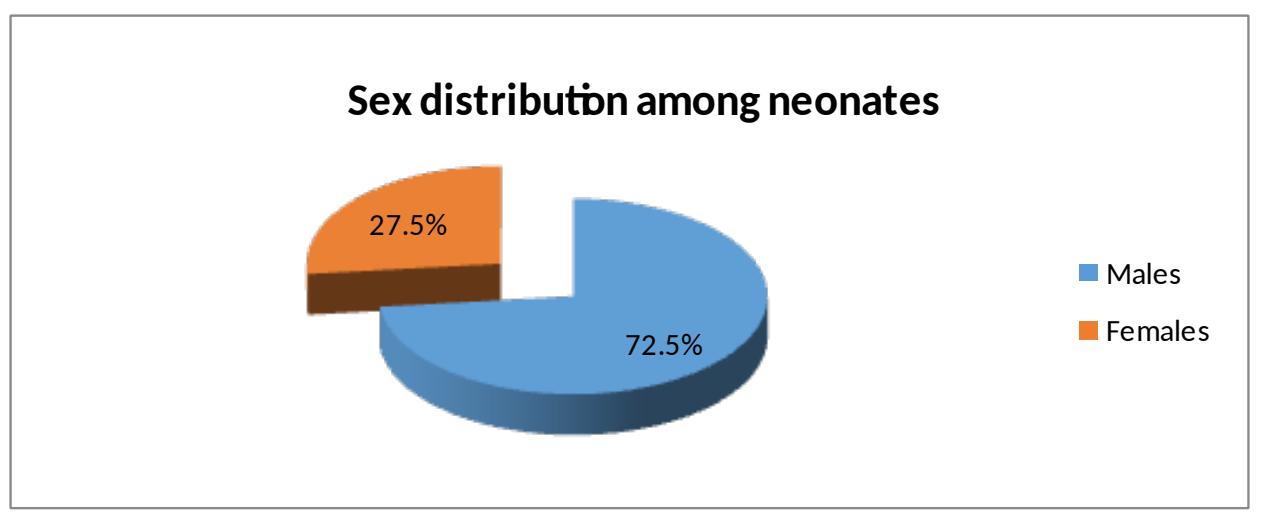

Figure (1): shows sex distribution among neonates under the study.

Table (2) showed mothers under the study that were free of diseases 17 (42.5\%) while 6(15\%), 7 $(17.5 \%), 6(15 \%), 2(5 \%)$ and $2(5 \%)$ were suffering from DM, pre-eclampsia, HTN, heart diseases and rheumatic diseases, respectively.

Table (2): Characteristics of mothers under the study:

\begin{tabular}{|l|l|l|}
\hline Characteristic & Number & Percent \\
\hline Medical co-morbidities of mothers: & & \\
No morbidity & 17 & $42.5 \%$ \\
DM & 6 & $15 \%$ \\
HTN & 7 & $17.5 \%$ \\
Pre-eclampsia & 6 & $15 \%$ \\
\hline
\end{tabular}




\begin{tabular}{|l|l|l|}
\hline Heart disease & 2 & $5 \%$ \\
Rheumatic disease & 2 & $5 \%$ \\
\hline Mode of delivery: & & \\
Normal vaginal delivery & 14 & $45 \%$ \\
CS & 26 & $55 \%$ \\
\hline Antenatal steroid given: & & \\
No & 9 & $22.5 \%$ \\
Yes & 31 & $77.5 \%$ \\
\hline
\end{tabular}

Table (3) shows that there is a significant association between mother comorbidity and positive cranial ultrasound findings. The $\mathrm{P}$ value equals $0.0405<0.05$.

Table (3): Relation between presence of mother comorbidity \& cranial ultrasound findings:

\begin{tabular}{|l|l|l|l|l|}
\hline Presence of mother co morbidity & Normal CUS & Abnormal CUS & Total & P value \\
\hline Normal mother & $7(17.5 \%)$ & $10(25 \%)$ & $17(42.5 \%)$ & \multirow{2}{*}{0.0405} \\
\cline { 1 - 4 } Co morbidity & $3(7.5 \%)$ & $20(50 \%)$ & $23(57.5 \%)$ & \\
\cline { 1 - 4 } Total & $10(25 \%)$ & $31(75 \%)$ & $40(100 \%)$ & \\
\hline
\end{tabular}

Table (4) reveals that the majority of the studied group (75\%) has abnormal US findings. While (25 $\%)$ with normal US findings.

Table (4): Percentage distribution of the studied group regarding US Findings:

\begin{tabular}{|l|l|l|}
\hline US findings & $\mathrm{No}(40)$ & Percent $(100.0 \%)$ \\
\hline Normal & 10 & $25.00 \%$ \\
\hline Abnormal & 30 & $75.00 \%$ \\
\hline
\end{tabular}

Table (5): The number of patients with each type of the brain lesion:

\begin{tabular}{|l|l|}
\hline Group & No. of patients \\
\hline Congenital & 5 \\
\hline acquired & 25 \\
\hline
\end{tabular}

Table (6): Findings in Ultrasound (congenital lesion):

\begin{tabular}{|l|l|l|}
\hline Congenital lesions & No. of patients & $\begin{array}{l}\text { Percentage in relation to total } \\
\text { studied neonates }\end{array}$ \\
\hline Dandy Walker & 1 & $2.5 \%$ \\
\hline Chiari malformation & 1 & $2.5 \%$ \\
\hline Germinal matrix cyst & 1 & $2.5 \%$ \\
\hline Arachnoid cyst & 1 & $2.5 \%$ \\
\hline Cavum septum pellucidum & 1 & $2.5 \%$ \\
\hline
\end{tabular}

Table (7): Finding in Ultrasound (Acquired lesion):

\begin{tabular}{|l|l|l|}
\hline U.S. Findings & No. of patients & $\begin{array}{l}\text { Percentage in relation to total } \\
\text { studied neonates }\end{array}$ \\
\hline Hypoxic ischemic encephalopathy (HIE) & 10 & $25 \%$ \\
\hline IVH grade I & 3 & $7.5 \%$ \\
\hline IVH grade III & 1 & $2.5 \%$ \\
\hline
\end{tabular}




\begin{tabular}{|l|l|l|}
\hline Subdural hemorrhage & 1 & $2.5 \%$ \\
\hline Intracerebral hematoma & 1 & $2.5 \%$ \\
\hline PVL & 1 & $2.5 \%$ \\
\hline Hydrocephalus & 8 & $20 \%$ \\
\hline
\end{tabular}

Table (8): Percentage distribution of the studied group according to mode of delivery:

\begin{tabular}{|l|l|l|}
\hline Mode of delivery & $\mathrm{No}(40)$ & Percentage \\
\hline NVD & 8 & $20 \%$ \\
\hline CS & 32 & $80 \%$ \\
\hline
\end{tabular}

Table (8) shows that majority of the studied group (80\%) were delivered by cesarean section.

Table (9): Relation between Mode of delivery between normal \&abnormal ultra-sound findings:

\begin{tabular}{|l|l|l|l|}
\hline Mode of delivery & Normal CUS & Abnormal CUS & Total \\
\hline NVD & $5(12.5 \%)$ & $3(7.5 \%)$ & $8(20 \%)$ \\
\hline CS & $5(12.5 \%)$ & $27(67.5 \%)$ & $32(80 \%)$ \\
\hline Total & $10(25 \%)$ & $30(75 \%)$ & $40(100 \%)$ \\
\hline
\end{tabular}

This table reveals that among those neonates delivered by CS, there is more percentage of abnormal US findings $(67.5 \%)$ vs $(12.5 \%)$. While among neonates that delivered by NVD there is more percentage of normal US findings $(12.5 \%)$ vs $(7.5 \%)$.

Table (10): Distribution of ultrasound findings within the abnormal studied group according to the maturity of the neonates:

\begin{tabular}{|l|l|l|}
\hline US findings & Preterm neonates & Full term neonates \\
\hline IVH Grade I & $3(7.5 \%)$ & $0(0.0) \%$ \\
\hline IVH Grade III & $1(2.5 \%)$ & $0(0.0) \%$ \\
\hline Subdural hemorrhage & $0(0.0) \%$ & $1(2.5 \%)$ \\
\hline Intracerebral hematoma & $0(0.0) \%$ & $1(2.5 \%)$ \\
\hline PVL & $1(2.5 \%)$ & $0(0.0) \%$ \\
\hline HIE & $0(0.0) \%$ & $10(25 \%)$ \\
\hline Hydrocephalus & $0(0.0) \%$ & $8(20 \%)$ \\
\hline Congenital lesions & $0(0.0) \%$ & $5(12.5 \%)$ \\
\hline
\end{tabular}

This table illustrates that all cases of IVH grade I ( 3 cases)(7.5\%), all cases of IVH grade III (1 case) $(2.5 \%) \&$ all cases of PVL ( 1 case) $(2.5 \%)$ were found in preterm neonates. While all cases of HIE(10cases) (25\%), all cases of hydrocephalus ( 8 cases(20\%), all cases of intracerebral hematoma(1 case)(2.5\%), all cases of subdural hemorrhage(1 case)(2.5\%)\&all cases with congenital lesions (5 cases) $(12.5 \%)$ were found in full term neonates.

Table (11): Frequency of trans-cranial Doppler diagnosis among neonates under the study:

\begin{tabular}{|l|l|l|}
\hline RI & Number & Percent \\
\hline Normal & 26 & $65 \%$ \\
\hline Decreased RI & 6 & $15 \%$ \\
\hline Increased RI & 8 & $20 \%$ \\
\hline
\end{tabular}




\begin{tabular}{|l|l|l|}
\hline Total & 40 & $100.0 \%$ \\
\hline
\end{tabular}

Table (11) showed that $26(65 \%)$ from the total 40 case were of normal RI, $6(15 \%)$ were decreased RI\&8 (20\%) were increased RI.

Table (12): Relation between trans-cranial Doppler and diagnosis by Ultra-Sound:

\begin{tabular}{|l|l|l|l|l|l|}
\hline & & Normal RI & Increased RI & Decreased RI & Total \\
\hline \multirow{4}{*}{$\begin{array}{l}\text { U.S } \\
\text { diagnosis }\end{array}$} & Normal & $10(25 \%)$ & $0(0.0 \%)$ & $0(0.0 \%)$ & $10(25 \%)$ \\
\cline { 2 - 6 } & HIE & $2(5 \%)$ & $2(5 \%)$ & $6(15 \%)$ & $10(25 \%)$ \\
\cline { 2 - 6 } & Hydrocephalus & $2(5 \%)$ & $6(15 \%)$ & $0(0.0 \%)$ & $8(20 \%)$ \\
\cline { 2 - 6 } & $\begin{array}{l}\text { Intracranial } \\
\text { hemorrhage }\end{array}$ & $6(15 \%)$ & $0(0.0 \%)$ & $0(0.0 \%)$ & $6(15 \%)$ \\
\cline { 2 - 6 } & Congenital lesions & $5(12.5 \%)$ & $0(0.0 \%)$ & $0(0.0 \%)$ & $5(12.5 \%)$ \\
\hline
\end{tabular}

Table (13): Relation between Doppler findings in normal patients and abnormal patients.

\begin{tabular}{|l|l|l|l|l|}
\hline & Normal patient & Abnormal patient & Total & P value \\
\hline Normal Doppler & $10(25 \%)$ & $16(40 \%)$ & $26(65 \%)$ & \multirow{2}{*}{0.0216} \\
\cline { 1 - 4 } Abnormal doppler & $0(0.0 \%)$ & $14(35 \%)$ & $14(35 \%)$ & \\
\cline { 1 - 3 } Total & $10(25 \%)$ & $30(75 \%)$ & $40(100 \%)$ & \\
\hline
\end{tabular}

The $\mathrm{P}$ value equals 0.0216 . The association between rows (groups) and columns (outcomes) is considered to be statistically significant.

Table (14): Relation between Doppler findings in normal cases and acquired lesions:

\begin{tabular}{|l|l|l|l|l|}
\hline & Normal patient & acquired lesions & Total & P value \\
\hline Normal doppler & $10(25 \%)$ & $11(27.5 \%)$ & $21(52.5 \%)$ & \multirow{2}{*}{0.0038} \\
\cline { 1 - 4 } Abnormal doppler & $0(0.0 \%)$ & $14(35 \%)$ & $14(35 \%)$ & \\
\cline { 1 - 4 } Total & $10(25 \%)$ & $25(62.5 \%)$ & $35(87.5 \%)$ & \\
\hline
\end{tabular}

The association between normal and abnormal Doppler in normal neonates and neonates with acquired lesions is considered statistically significant. $\mathrm{P}$ value equals 0.0038 . 
acquired lesions. So, we divided the abnormal :group into two subgroups Subgroup (A) includes the congenital lesion • cases: Dandy Walker (2.5\%), Chiari malformation $(2.5 \%)$, germinal matrix cyst (2.5\%), arachnoid cyst (2.5\%), cavum septum .pellucidum $(2.5 \%)$

Subgroup (B) include the acquired lesion • cases: Hypoxic ischemic encephalopathy (HIE) 10 patients $(25 \%)$, IVH grade I 3 patients $(7.5 \%)$, IVH grade III one patient $(2.5$ $\%$,subdural hemorrhage one patient(2.5\%), intracerebral hemorrhage one patient(2.5\%),PVL one patient $(2.5 \%) \&$

.Hydrocephalus 8 patients $(27.5 \%)$ In a study conducted by Ziaul and his colleagues (8) upon 103 preterm neonates, 56 (54.3\%) had normal CUS findings and 47(45.63\%) had abnormal CUS findings. Among the abnormal CUS findings, 22 (21.4\%) had cerebral edema, 17 (16.5\%) had .IVH and $8(7.8 \%)$ had ventricular dilatation This difference arises because they studied only premature neonates as they are high-risk group for intracranial lesions but we studied premature and full term neonates with clinical signs of neurological disorders because we thought that there are many cases with intracranial lesions in full term as preterm .neonates

Neonatal intracranial hemorrhagic and hypoxic lesions can be divided as those

\section{Discussion .4}

Cranial ultrasound is considered the most available and easily repeatable technique for imaging the neonatal brain in both the preterm and full term infants. Its diagnostic accuracy depend on various factors; the suitability of the ultrasound machine for neonatal cranial work, the use of optimal settings and probes, appropriate scanning protocols, the use of a variety of acoustic windows ,the scanning experience of the examiner. Besides, not least, knowledge of normal anatomy and the echogenicities of different tissues in normal .and pathological situations (7)

We designed a cross sectional study on 40 patients in neonates group with definite or suspected neurological lesion based on clinical .examination. All cases had cranial ultrasound We divided our patients into two groups according to CUS diagnosis: normal CUS .group and abnormal CUS group

The purpose of this study is to document the value of neonatal transcranial ultrasound and transcranial Doppler as a method of diagnosis of brain pathologies such as hemorrhagic, ischemic \& congenital anomaly .events in neonates

This study showed that the Ultra-sound diagnosed $10(25 \%)$ with normal brain and 30 (75\%) with abnormal CUS findings. Among the abnormal CUS findings, 5(16.6\%) with congenital abnormalities, 25(83.3\%) with 
This result coincide with the study of Malloy (12) who stated that among infants delivered at 32 to 36 weeks gestation, an increased risk for neonatal mortality and morbidity exists among those delivered by primary cesarean section compared with vaginal delivery. The mechanism by which the cesarean section increases the risk for neonatal mortality is not clear, but the particular morbidities examined suggest that the mechanism, at least for the morbidities, may be related to the impact of cesarean section on .respiratory adaptation This result may contradict the study conducted by Elisa and her coworkers (13) who stated that there was no significant association between abnormal cranial ultrasonography and the mode of delivery The study of Henrietta and his workmates (14) stated that Doppler flow measurements may help to distinguish between vascular structures and non-vascular lesions and CCD can be applied to study cerebral hemodynamics through assessing the major intracranial .arteries as well as the large veins In our study, Transcranial color-coded Doppler was especially applied to assess vascular lesions in all examined patients to measure RI of ACA or MCA. We found abnormal RI in acquired lesions including HIE\& .hydrocephalic patients occurring in the preterm \&full term neonates. In the preterm, the major lesions are germinal matrix hemorrhage $(\mathrm{GMH}) /$ intraventricular hemorrhage (IVH) and periventricular leucomalacia (PVL) which is similar to those .found by Laura and his coworkers (9) In the term infants the major problems are hypoxic-ischemic encephalopathy/injury (HII) and intracranial hemorrhage. Which is similar .to those found by Gupta (10) Intracranial hemorrhage is uncommon in term infants and when it occurs is generally unrelated to the germinal matrix hemorrhage, which is similar to those found by Siu and his .collaborators (11) In our study, we found that number of male patients exceeded that of females being 29 male patients and 11 female patients representing $72.5 \%$ and $27.5 \%$ of all patients respectively. However the significance of this .finding is questionable In order to detect the comorbidities associated with abnormal ultrasound findings, the study reveals significant association between abnormal TCUS and mode of delivery as the neonates delivered by cesarean section (CS) have a higher percentage $(27 / 32,84.3 \%)$ of abnormal ultrasound finding compared to those born with NVD who have higher percentage $(5 / 8,62.5 \%)$ of normal .ultrasound findings 
cranial ultrasonography should be performed in all neonates admitted to a neonatal intensive

.care unit

\section{Conclusion and Recommendations .5}

US has been a major advance in the study of neonatal brain, it is portable safe, noninvasive, low cost and highly effective technique that is of considerable value in evaluation of neonatal intracranial disorders and should be included within integrated .approach to CNS imaging in the neonates

\section{References}

1. North $\mathrm{K}$ and Lowe L. Modern head ultrasound: Normal anatomy, variants, and pitfalls that may simulate disease. Ultrasound Clin., 2009; 4: 497-512.

2. Huang $B$ and Castillo M. Hypoxicischemic brain injury: Radio Graphics, 2008; 28: 417-439;

3. Couture A and Veyrac C. Transfontanellar Doppler imaging in neonates, $1 \mathrm{st}$ edn. 2001; Springer, Berlin

4. Nicoletto HA and Boland LS. Transcranial Doppler series part V: specialty applications," American Journal of Electroneurodiagnostic Technology, 2011; 9 (51)1: 31-41.

5. Epelman M, Daneman A and Kellenberger C. Neonatal encephalopathy: a prospective comparison of head US and MRI. Pediatr Radiol., 2010; 40:1640-1650.
Our study declared that HIE was more .associated with decreased or increased RI This results are confirmed with the results made by Liu and his workfellows (15) who stated that in HIE groups, the blood flow velocities decreased or increased markedly according to the degree of cerebral hemodynamic disturbance, RI increased or .decreased accordingly

Our study showed that hydrocephalus was .more associated with increased RI This result is supported by Kolarovszki and his associates (16) who revealed that hydrocephalus negatively affects the cerebral blood flow. End-diastolic blood flow velocity .is decreased and resistive index increased CCD and TCD were very beneficial in detecting vascularity of the lesions and assessment of the intracranial vessels: for example, it was used to study the vascularity of brain lesions and to assess the increased intracranial tension in the hydrocephalic patients which is similar to those found by

.Henrietta and his colleagues (14) American Academy of Neurology and the Practice Committee of Child Neurology recommended routine cranial ultrasonography screening on all newborns born before 30 weeks of gestational age. The Canadian Pediatric Society suggests the need for cranial ultrasonography before 32 weeks of gestational age. According to Meijler (17), 
United States, 2000-2003. Birth, 2009; 36: 26-33.

13. Elisa B, Anna $\mathrm{T}$ and Alessandro $\mathrm{B}$. Universal Cranial Ultrasound Screening in Preterm Infants with Gestational Age 3336 Weeks. A Retrospective Analysis of 724 Newborns. Pediatric Neurology, 2014; ( 51 ):790-794

14.Henrietta KR, Vijay V and John A. Pediatric brain. In: John P. Mc Gahan, Barry B. Goldberg (eds). Diagnostic ultrasound, 2nd edn. Vol.2. Informa Healthcare: New York-London, 2008; 563-612.

15. Liu J and Cao HY. Ultrasound diagnosis of neonatal hypoxicischemic encephalopathy. In: New trends in brain hypoxia ischemia research. Edited by: Hamalainen E. Nova science publishers, 2008; (10):251-261.

16.Kolarovszki $\mathrm{B}$ and Zibolen $\mathrm{M}$. Transcranial Doppler Ultrasonography in the Management of Neonatal Hydrocephalus, Hydrocephalus, Dr Sadip Pant (Ed.), ISBN, 2012; 978-953.

17.Meijler G. (Timing of ultrasound examination. In: Neonatal Cranial Ultrasonography. Guidelines for the Procedure and Atlas of Normal Ultrasound Anatomy. 2nd ed. Springer-Vergalg Heideberg, 2012; 3: 57-73.
6. Griffiths P, Paley $M$ and Whitby E. Imaging the central nervous system of the fetus and neonate. Taylor and Francis Group Publishers, ISBN-2006; 13: 978-08247-2856-4, New York, USA.

7. Lara M, LeijserLinda S, de VriesFrances M. Using cerebral ultrasound effectively in the newborn infant Early Human Development, 2006; 82(12): 827-835.

8. Ziaul M, Atiqul I, Monir H and Naila Z. Cranial Ultrasound in Preterm Neonates: Screening of at Risk Infants for Long Term Neurodevelopmental Impairments. Journal of Enam Medical College, 2016; 6(1): 2832

9. Laura $\mathrm{R}$ and Keller S. Intraventricular Hemorrhage of preterm neonate. In: Kenneth FS, Stephan A, editors. Pediatric Neurology. ed 3. 1999; Vol 1. Mosby; pp. 205-219.

10.Gupta A. Hypoxic-Ischemic Encephalopathy. In: Berry M, Suri S, Chouwdhry V, Chouwdhry JP, editors. Diagnostic Radiology: Pediatric Radiology, 1997; pp. 260-273.

11.Siu Y, Kwong NS and So K. A 10-year review of intracranial hemorrhage in term neonates. HK J Pediatr., 2006; 11:140146.

12. Malloy M. Impact of cesarean section on intermediate and late preterm births: 

